\title{
A philosophical analysis on the challenge of cultural context to Al translation
}

\begin{abstract}
Language translation is a fast growing field of Artificial Intelligence, which helps people to learn and better understand other cultures different from their own. Although currently the quality of AI translation is still far less than satisfactory compared with experienced human translators, it is under steady improvement. Correspondingly, the concern over the limit of the quality improvement of AI translation would naturally arise, in a sense similar to the concern over the limit of the efficiency improvement of heat engines a couple centuries ago. Obviously, this would not be a pure technological issue, but an essentially philosophical issue due to the cultural openness of the human language system.
\end{abstract}

As is pointed out in this writing, the biggest challenge to AI translation is the complexity of the cultural context of the original text in general, instead of the textual context within the text. Meanwhile, the currently existing knowledge pool of human culture which is accessible to AI machines appears very poor, because the history of AI is still very short and the team of AI scientists is quite small while the human cultural history is very long and the size of the human cultural domain is enormous. Therefore, the major task for the development of AI translation, in terms of dealing with the cultural context, is to catch up with the continually evolving and highly diversified human cultural environment around the world. For this reason, this writing would focus on the comparison between the advantages and disadvantages of humans and AI machines in terms of learning the culture.

Keywords: thermal dynamics, AI translation, philosophical, vocabulary, diversified
Volume 5 Issue 4 - 2019

\author{
Rongqing Dai \\ Freelance philosopher, New Jersey, USA
}

Correspondence: Rongqing Dai, freelance philosopher, New Jersey, USA, Tel 00I-732-395-3163, ORCID 0000-0003-1926930I, Email rongqing.dai.mail@gmail.com

Received: August 14,2019 | Published: August 30, 2019

\section{Introduction}

AI translation technology is a sweet blessing for many who need to understand or express ideas in multiple languages. However, for those users who are proficient in the source and the target languages, using AI translators to help improving the quality of their writings could be a very frustrating matter, especially with long paragraphs, since it would be too easy and too often for them to find errors in the translations.

It is a common phenomenon with AI translation that errors in a translation can be reduced or even removed simply by truncating the source paragraph bit by bit until no errors left in the translation or no further truncation can be made to the source paragraph. That is to say, a sentence could be translated wrong when being put into a long paragraph, while the same sentence is correctly translated when being input as an atomic single sentence or being in a short paragraph. Or we may say the easiest way to have an AI translator make mistakes is to give it a long paragraph. The direct reason behind this symptom is quite simple: for a simple sentence such as "the water here is very deep", when given as an atomic sentence without any context, the meaning is universally clear and definite, and thus it would be very easy for the translator to give the answer in any target language. But once the sentence is put into a complicated paragraph, the $\mathrm{AI}$ translator would need to worry about the influence of its context upon the sentence, as well as the influence of that sentence upon others as part of their context.

Based on the common knowledge of how computers work according to the binary code installed on their hardware, it is philosophically clear, even without the involvement of technological details, that when given a paragraph, no matter how obvious the meaning of each sentence and the meaning of the whole paragraph is to a human reader, the AI translator needs to go through the same preset rules of analyzing the contextual relationship and the mutual influences between the sentences; otherwise, AI translators would never be able to handle anything that is a bit more complicated than a propositional statement such as "I am a man." During the process of identifying the contextual relationships, a critical challenge would be the cultural complexity induced by the openness of natural language. Accordingly, as we might find in this article that the main challenge for AI translation does not come from the textual context of each sentence, but from the cultural context of the whole text, and thus this is not a simple technical challenge, but a profound philosophical issue which is meaningful to AI translation or AI learning.

\section{The challenge of cultural context}

Human culture is an extremely complex system, which is dynamically related to the environment and the general custom of each relatively independent community. Due to the extremely large number of dimensions of a cultural system in general, it would be much harder to learn a completely open cultural system than to master the rules (as well as the logic of how to play with the rules) of a closed scope game such as chess, Go, or even Jeopardy ${ }^{1}$ for which a limited vocabulary is exploited in a discrete manner.

Besides, the dynamics of human culture is always linguistically reflected in the dissimilarities between the diverse forms of language presentation across the world, as well as the subtly varying varieties of the same language for different communities. It is noticed by many 
people that the customary usage or pronunciation of certain English words could be changed in different cities or even neighboring towns in the same country. In this sense, natural languages do not strictly follow the grammatical rules in the same way as computers would follow the coding language syntaxes, but dynamically evolve as life goes on in every community which is more or less different from the rest of the world.

Consequently, tackling the overall lexicon complexity would be an extremely daunting task due to the seemingly countless varieties of languages and dialects around the world; however, so far we don't see any insuperable logical obstacle to make it impossible for AI scientists to accomplish that task, in the way that the Thermal Dynamics Second Law would prohibit anyone from making a perpetual motion machine. Although some technical issues such as the language ambiguities, ${ }^{2}$ non-standard usage of words, named entities, or even special community slangs would create great difficulties for AI scientists to battle with, they might still possibly be all resolved by the combination of advanced pattern recognition technology (plus sufficiently great computational power) and the diligent human laboring, when enough capital would be invested in the field.

In fact, as we all know, computers do have the advantages over humans with many things, especially the enormously huge memory and extremely rapid processing speed, which enable them to process complicated big data even in real time; besides, they will never forget whatever they have learned collectively unless some severe catastrophe would happen to this globe. All these advantages would make them very potent for solving issues that might pose the quantitative obstacles to humans due to their large sizes. In addition to these hardware advantages of computers, we ought not to ignore the importance of human efforts in the expansion of the lexicon storage for AI machines either. There is no physical law to nullify the possibility for someone to invest a huge amount of capital for expanding the lexicon storage of AI translation so as to have a representative from every community in the world working on the project, no matter how practically impossible it might sound. This lack of physical impossibility could imply some kind of socially practical potentiality, no matter how small it might be. In the case of the non-standard usage of words, as an example, ideally, as long as the lexicon storage could be expanded to large enough (by making use of the above mentioned hardware advantage and human resources), so that all known varieties of usage in most commonly occurring textual contexts of any word in any language are collected in the storage, then we would practically have satisfactory translations for all cases of the non-standard usage of words.

Therefore, unless it is mathematically proved that it would be impossible for AI scientists to resolve the above mentioned technical obstacles to AI translation (which are essentially various matters of quantitative difficulties), even with all the potentially possible computer power and human efforts being exhausted, we might still reasonably expect that those technical obstacles might no longer be valid some day when AI technology and computer power are much more advanced. This awareness could positively help to set our expectation about to what extent AI translation might evolve in the coming years, as the overall AI technology and computer power advance with lightning speed.

However, we might also find some fundamentally irresolvable hurdles to the business of AI translation through philosophical analysis, even without involving any technical details; accordingly, it would be beneficial for AI scientists to distinguish them from those potentially solvable technical obstacles before they set off to improve the quality of AI translation.

\section{A fundamental difference between humans and Al}

Philosophically speaking, the most fundamental difference between humans and AI machines in terms of learning human culture is that we are living in the cultural system, but AI machines are learning about the system that is completely disconnected from their being, which makes us the insiders and them the outsiders. This difference is not as trivial as it might sound like because it gives us a huge advantage that we could learn things while we are not intentionally learning.

For humans, the most important part of learning is to understand, as Searle correctly pointed out in his Chinese Room thought experiment ${ }^{3}$ when responding to the abstract propositions of thinking machines. ${ }^{4}$ Human understanding of things in real life would be highly tied up with the cultural preparation of the subject who attempts to understand, as Wittgenstein once famously said, "If a lion could talk, we could not understand him."

Although AI machines don't really need to "understand" our culture to interact with us, as Searle demonstrated, it would be necessary for them to have a good knowledge about the relationships that are meaningful in the culture so as to effectively interact with us in our cultural environment, which subtly or radically varies from place to place around the world, and rapidly evolves from time to time along history. Correspondingly, the fact that we learn our culture while we are living in the culture but AI machines need to learn our culture as outside observers would undoubtedly become a huge challenge to AI machines, or more precisely, to the human designers of AI machines. But on the other hand, AI machines do have their natural advantage as well: the collectively accumulative learning. Everything that all AI machines have learned so far or will learn in the coming future, as well as everything that AI scientists have put into their databases as part of the knowledge accessible to AI machines, could be virtually shared among all AI machines if integrated together into a central database that is accessible to all AI machines. Therefore, virtually, AI machine learning is one for all and all for one.

Contrary to machine learning, not a single human being can learn things for others, and no human mind can retrieve knowledge in the head of another person without learning; furthermore, all the knowledge that each person learns ends at the moment when the life of that person ends, and the new knowledge humans are learning now could soon become part of the learnt contents of AI machines (at least with the help of AI scientists, in case AI machines are not mature enough).

Therefore, while the ultimate obstacle for AI machines to learn human culture is the complexity of human culture and the fact that $\mathrm{AI}$ machines cannot learn the culture in the way (the easy way) as humans, the main advantage for AI machines is their quickly evolving and irreversible collective accumulation of knowledge. Hence, the quality of AI translation would be determined by how much the extremely rapid collective accumulation of the knowledge about human culture by AI machines can help to offset their innate deficiency in learning human culture. 


\section{Main philosophical obstacles for Al learning}

There are a few cultural domains, for which AI translation would never closely match human translation. First, when it comes to the domain of human mind and body, AI machines have to learn everything from human descriptions, despite the fact that it is impossible for humans to describe our own internal experiences or feelings in precise details. Therefore, it is basically impossible for AI machines to fully match humans in learning certain human cultural activities that are directly related to human mind and body domain, such as meditation, mutual loving, and so on, no matter how hard their human designers would try to present the relevant knowledge to AI machines in writing. Correspondingly, it would be impossible for AI machines to fully match humans when translating text about those activities. But on the other hand, since the basic way for humans to understand each others' feelings is through the imprecise language expressions, which often cause misunderstandings, the imperfection of machine translation as a result of copying human descriptions without sensing the real human feelings might be tolerable to a certain extent.

Secondly, it is not always possible to find exact simple phrases of wording in the target language to match the phrases in the source language, and thus human translators often need to create some complicated expressions based on their personal understandings, which would often involve some linguistic implications that do not appear in the original paragraph of the source language, and sometimes even body language or facial expression. Since AI translation is based on their previously recorded data, it would be extremely difficult for them (or their designers) to solve this problem of the nonexistence of matching phrases.

Thirdly, even if AI machines can manage to match a simple phrase in the source language with a combination of complicated words in the target language, through very powerful pattern recognition algorithm in the future, it would be impossible for AI machines to communicate with humans through meaningful body language or facial expression as the human speakers do, no matter how advanced facial recognition technology is employed with the help of extremely high quality cameras and fancy artificial skins from the nanotechnology. This is because AI machines cannot sense the subtlety of the inner feelings of the human speakers.

Fourthly, as for linguistic implications, AI machines would not need to use implication if they do know what they need to say since AI machines do not speak based on feelings but based on context matching; on the other hand, it would be disastrous if AI machines start to proactively use implications when they don't know what they are implying. Therefore, linguistic implications would be a forbidden territory for AI machines, no matter for translation or for future machine-human communication.

Since these philosophically meaningful difficulties cannot be simply resolved through computer programming techniques, they are entitled as philosophical obstacles in this writing.

\section{More advantages of Al machines}

As mentioned earlier, computers do have many technical advantages in general. For AI translation, all those general advantages are especially meaningful in terms of the fast update of their knowledge base when learning human culture. Since it is technically viable to have AI machines to learn from their own mistakes through human reactions or through manual corrections by AI scientists, with the above mentioned technical advantages, AI machines would be capable of quickly updating their knowledge in order to adapt to various cultural environments. This might be of critical importance when it comes to the issue of whether the rapid knowledge accumulation by $\mathrm{AI}$ machines as a whole would be fast enough to enable them to catch the continually evolving human culture, even with their disadvantage of a late start in the game.

In addition to all the above advantages, the fact that AI machines do not share human feelings, which makes them philosophically disadvantageous in understanding human culture, would actually also be an important advantage for them in the sense that they would not be distracted by the illogical feelings as humans would.

\section{Conclusion}

Because of the fundamental difference that exists in the course of learning culture by humans and by AI machines, cultural context in general would be an insurmountable hurdle for AI translation, no matter how intelligently the translating software is programmed, how much advanced math is involved, and how much computational power is utilized. It is just philosophically impossible for AI machines to be as good as human translators when it comes to understanding human culture, even though their collective mastery of vocabulary could be potentially much better than different individual human translators. The most fundamental reason of this is because we are living in the culture but they are learning from our expressions, as the outsiders of the culture, while a large part of human communication is based on the mutual understanding of the culture without the need of explicit language expression, especially when aided with body language and facial expression plus various types of understandable linguistic implications. Besides, human culture in general is extremely complicated, with subtle or radical variations across different cultural communities, and ceaselessly evolves in time, which will undoubtedly make AI translation an extremely daunting task, even though the complexity of the contents alone might not be a logically irresolvable problem in many cases.

\section{Acknowledgments}

None.

\section{Conflicts of interest}

The author declares there are no conflicts of interest.

\section{Funding}

None.

\section{References}

1. https://www.theguardian.com/technology/2011/feb/17/ibm-computerwatson-wins-jeopardy.

2. Hillel YB. The Present Status of Automatic Translation of Languages. Advances in Computers. 1960;1:91-163.

3. Searle JR. Minds, brains and programs. Behavioral and Brain Sciences. 1980;3(3):417-457.

4. Turing AM. Computing Machinery and Intelligence. Mind. 1950;49:433460 .

5. Wittgenstein L. Philosophical Investigations. UK: Oxford, Basil Blackwell ltd; 1953. 